\title{
Experimental Studies in Rats on the Effects on Growth and Intestinal Microflora of a New Mix Combining Calcium, Iron, Zinc and a Prebiotic in a Milk-like Vehicle
}

\author{
F. C. Tesan ${ }^{1, *}$, E. Rubeglio ${ }^{3}$, E. Maciero ${ }^{4}$, H. Torti ${ }^{1}$, J. Orlandini $^{4}$, R. Weill ${ }^{4}$, J. Boccio $^{1}$ and \\ M. J. Salgueiro ${ }^{1,2}$ \\ ${ }^{I}$ Stable Isotope Laboratory Applied to Biology and Medicine, School of Pharmacy and Biochemistry, University of \\ Buenos Aires, Argentina \\ ${ }^{2}$ Radioisotope, Laboratory, Physics Department, School of Pharmacy and Biochemistry, University of Buenos Aires, \\ Argentina \\ ${ }^{3}$ Laboratory of Microbiological, Analysis, Buenos Aires, Argentina \\ ${ }^{4}$ Research and Development Department,Danone Argentina SA, Buenos Aires, Argentina
}

\begin{abstract}
The aim of this work was to determine the effects of a new mineral mix combined with a prebiotic on growth, mineral deposit and intestinal microflora using a milk-like vehicle. This study was performed with Sprague Dawley female rats separated into 7 groups. Group 1 was fed with a deficient diet on its mineral content, group 2, 3 and 4 were fed with normal diets on their mineral content during 28 days but group 2 was fed with a diet containing reference $\mathrm{Ca}, \mathrm{Fe}$ and $\mathrm{Zn}$ sources and groups 3 and 4 with diets containing other $\mathrm{Ca}, \mathrm{Fe}$ and $\mathrm{Zn}$ sources that were evaluated. Group 4 received additionally a diet containing a prebiotic. Groups 5,6 and 7 were submitted to a depletion period of 14 days during which they were fed as group 1 and then to a repletion period of 14 days when they were fed with the same diets as group 2, 3 and 4 respectively. Initial and final body weight, average growth rate, liver and femur weight, iron and zinc liver content as well as calcium and zinc femur content were the parameters evaluated for the growth and mineral deposit analysis. Anaerobes, enterobacteria, lactobacilli and bifidobacteria recount was performed from feces samples collected on different treatment days. Mineral sources under study presented slightly better characteristics than the reference standard ones. Moreover, the combination with a prebiotic may produce some advantages related to mineral absorption and intestinal microflora composition as a bifidogenic effect was confirmed.
\end{abstract}

Keywords: Prebiotic, calcium, iron, zinc, food fortification, Sprague- Dawley rats.

\section{INTRODUCTION}

Malnutrition is still a very relevant matter that affects children mostly in their early development ages. Severe consequences on growth, immune function, cognitive and sexual development are related to this affection. Strategies to overcome child malnourishment have been studied for many decades, and one of the most significant issues of this problem is mineral deficiency [1]. Some of the minerals that are essential for growth and physiological normal functioning are iron, calcium and zinc [2-5]. In general, the main but not the only cause of mineral deficiency is nutritional. Thus, mineral intake is lower than the needed and/or eating habits include components that inhibit some mineral absorption such as phytates present in vegetables [6]. However, previous works by many authors have shown that some of the devastating consequences that poor mineral consumption has on growth can be reverted by a repletion period in which normal mineral intake is restored [7-9]. In order to prevent these

*Address correspondence to this author at the Stable Isotope Laboratory Applied to Biology and Medicine, Physics Department, School of Pharmacy and Biochemistry, University of Buenos Aires, Argentina; Tel: (+5411) 49648202/8277, ext 33; Fax: (+5411) 4964-8202/8277, ext 33;

E-mail: ftesan@ffyb.uba.ar deficiencies, food fortification is a much valid option from several points of view related to economical and technological aspects of food processing and consumption. A change in peoples eating habits is not expected by this strategy: previous preferences and food habits should be contemplated. In this way, the mineral sources as well as the vehicle should be carefully chosen, considering the target consumer which can involve the entire population or just groups that are at high risk. Taste, smell and color of the fortified food should not be different from the unfortified one and it is recommended the addition of protective components so as to neutralize the negative effects in case of the presence of mineral inhibitors. Multiple mineral fortification tends to be more cost effective than single mineral one and related to this it can be mentioned that the fortified food should not exceed the final cost of the unfortified one $[10,11]$.

Over the past years, the evaluation of the beneficial effects of prebiotics has become an area of increasing interest. They have already been classified as products with several advantages for human's health but since further research is needed to support that information, they are still a frequent subject of study. Consequences of prebiotics intake, as polidextrose and fructooligosaccharides like inulin, involve 
an alteration of the intestinal microflora, mostly an increment in bifidobacteria and lactobacilli populations, resulting in a better functioning of the bowel. There are some positive effects that can be related to this, such as: an increment in some nutrients bioavailability like calcium $[12,13]$, an enhanced short chain fatty acid production resulting in a decrease in cholesterol levels, an anti-diarrheal effect, a decrease in blood glucose levels [14], a beneficial impact on triacylgricerids metabolism $[15,16]$, possible prevention from some diseases as cancer and the improvement of some of the immune system functions [17]. Some papers have also mentioned prebiotics as a potential solution for constipation in the elderly since they improve intestinal motility with a positive impact in transit time [18]. Foods such as artichokes, onions or garlic are natural prebiotics sources but supplementation of other food with components that are regarded as prebiotics like inulin, galactose polymers or different types of oligosaccharides is being considered. This would allow these products to reach people whose diets do not include the foods mentioned above or to provide a bigger prebiotic intake by adding another source. In this way, the effects on the host's intestinal microflora of the added prebiotic should be confirmed. On the other hand, and in a similar way that for fortified foods, the final product should not suffer modifications on its organoleptic characteristics and most important on the nutritional impact it has compared to when the prebiotic is not present. This should also be confirmed by scientific studies.

Supplemented foods can be considered as functional foods since they provide not only nutrition to the consumer but a physiological benefit too [19]. Functional foods have become very popular these days but before they reach the market several studies must be performed in order to confirm their additional benefit to people that consume them and to certify the health claims that appear on their labels.

Thus, the aim of this work is to analyze the impact on growth and intestinal microflora of a new mix combining calcium, iron, zinc and a prebiotic in a milk-like vehicle by means of comparative studies in rats.

\section{MATERIALS AND METHODOLOGY}

\section{Animals}

All animal experiments were performed in accordance with the "Guide for the Care and Use of Laboratory Animals, U.S. National Research Council, 1996". We used 70 female Sprague Dawley weaned rats (School of Veterinary, University of Buenos Aires, Argentina) that were housed in individual stainless steel cages with grated floor and collec- tion trays of the same material. Rats were kept in $12 \mathrm{~h}$ light followed by $12 \mathrm{~h}$ darkness cycles and in humidity and temperature controlled room. All diets and distilled water were provided ad libitum to them.

\section{Reagents Used for the Preparation of the Experimental Diets}

The mineral sources used for the preparation of the diets were $\mathrm{FeSO}_{4} \cdot 7 \mathrm{H}_{2} \mathrm{O}$ (Lipotech, Argentina), $\mathrm{ZnSO}_{4} \cdot 7 \mathrm{H}_{2} \mathrm{O}$ (JT BAKER, Mexico), and $\mathrm{Ca}_{3}\left(\mathrm{PO}_{4}\right)_{2}$ (Prayon INC., USA) as reference standards (see below diet NS). On the other hand, ferrous gluconate stabilized with glycine (Lipotech, Argentina), zinc gluconate stabilized with glycine (Lipotech, Argentina) and tricalcium phosphate stabilized with glycine (Lipotech, Argentina) were used as the mineral sources under study for the combination with the prebiotic in the milk-like vehicle (Danone Argentina SA, Argentina), see below diets NE and NEP. The prebiotic under evaluation was a combination of inulin, polidextrose and arabig gum (see below diet NEP).

\section{Diets}

Four different diets were used in the protocol and except for one of them (see below, diet D) their macro and micronutrient final contents were according to the recommendations of the AIN-93G for laboratory rodents [20]. The first diet coded as D was prepared as deficient in its iron $(5.7 \mathrm{ppm})$, zinc $(6.1 \mathrm{ppm})$ and calcium $(170 \mathrm{ppm})$ content, which were provided directly in the mineral mix. The other three diets were also prepared as described in the AIN-93G, but the mineral sources such as iron, zinc and calcium were separately provided in a milk-like vehicle $(63 \%$ water, $30 \%$ milk, $6 \%$ sugar, and $1 \%$ milky cream) added at $10 \%$ of the final diet. Thus the second diet coded as NS was prepared using the reference standard mineral sources added in the milk-like vehicle; the third diet coded as NE was prepared using the mineral sources under study added in a milk-like vehicle and the forth diet coded as NEP was prepared using the mineral sources under study and the prebiotic added at $1 \%$ in a milklike vehicle. Diets mineral contribution as well as their composition are shown in Tables 1 and 2 respectively.

\section{Treatments}

Animals were randomly divided in 7 groups of 10 rats each. Group 1 was fed with D diet, Group 2 with NS diet, Group 3 with NE diet and Group 4 with NEP diet during 28 days. Groups 5, 6 and 7 were submitted to a depletion period of 14 days during which they were fed with $\mathrm{D}$ diet and then

Table 1. Contribution of Mineral Elements (Ca, Fe and Zn) to the AIN- 93G and Experimental Diets

\begin{tabular}{|c|c|c|c|c|c|}
\hline & \multirow{2}{*}{ AIN-93G Recommendation } & \multicolumn{4}{|c|}{ Experimental Diets } \\
\cline { 3 - 6 } & & Diet D & Diet NS & Diet NE & Diet NEP \\
\hline \hline $\mathrm{Ca}(\mathrm{g} / \mathrm{Kg}$ diet $)$ & 5 & $0.17 \pm 0.01$ & $5.6 \pm 1.0$ & $5.6 \pm 1.0$ & $5.6 \pm 1.0$ \\
\hline $\mathrm{Fe}(\mathrm{mg} / \mathrm{Kg}$ diet $)$ & 35 & $5.7 \pm 0.5$ & $35.0 \pm 1.0$ & $34.6 \pm 1.0$ & $34.0 \pm 4.1$ \\
\hline $\mathrm{Zn}(\mathrm{mg} / \mathrm{Kg}$ diet $)$ & 30 & $6.1 \pm 2.9$ & $30.2 \pm 8.6$ & $38.0 \pm 9.3$ & No \\
\hline Prebiotic & ---- & No & No & Yes \\
\hline
\end{tabular}


Table 2. Experimental Diets Composition

\begin{tabular}{|l|ll|}
\hline Diet D & $\bullet$ & $100 \%$ diet base AIN-93G deficient in Ca, Fe and Zn \\
\hline Diet NS & $\bullet$ & $\begin{array}{l}90 \% \text { diet base AIN-93G } \\
10 \% \text { vehicle fortified with Ca, Fe and Zn reference sources }\end{array}$ \\
\hline Diet NE & $\bullet$ & $\begin{array}{l}90 \% \text { diet base AIN-93G } \\
10 \% \text { vehicle fortified with Ca, Fe and Zn sources under study }\end{array}$ \\
\hline Diet NEP & $\bullet$ & $\begin{array}{l}90 \% \text { diet base AIN } 93 G \\
10 \% \text { vehicle fortified with Ca, Fe and Zn sources under study and prebiotic (1\% of vehicle). }\end{array}$ \\
\hline
\end{tabular}

to a repletion period of 14 days during which they were fed with NS, NE and NEP diets, respectively.

Food intake was noted daily and body weight was recorded three times a week for each group in order to register the effects of the treatment on growth.

The bacteriological research involved collection of feces samples which were taken on day 0 (basal), day 14 (second sample) and day 28 (third sample) starting from the beginning of the treatment with experimental diets. After this first period of 28 days, all groups were fed with a balanced diet (Ganave alimentos balanceados, Argentina) and two additional feces samples were taken on days 30 and 40 of the protocol. This means that samples on days 0,14 and 28 are related to the effect of diets under study and samples of day 30 and 40 to the "wash out" effect of the balanced diet.

Finally, on day 40, animals were sacrificed by cervical dislocation after being anesthetized in order to get samples of liver and femur for evaluation of mineral deposits of iron and zinc, and zinc and calcium, respectively.

Bacteriological analysis was performed in order to determine: 1) total enterobacteria; 2) total anaerobes; 3) lactobacilli and 4) bifidobacteria.

\section{Samples Processing}

Feces samples were collected directly from the rectum of each rat maintaining aseptic conditions and avoiding their contact with urine. Samples were kept and transported in anaerobiosis (GEN bag bioMerieux, France) until coprocultive were performed.

Each sample represented the feces from 10 animals. It was obtained mixing equal parts of each animal feces up to one gram. Each pool was dissolved in $9 \mathrm{ml}$ of Ringer/cystein $(0.3 \mathrm{~d} / \mathrm{l})$ solution. From this, successive decimal dilutions were made and $0.1 \mathrm{~mL}$ of them were inoculated in the following culture media: for total enterobacteria, Mac Conkey Agar (Britannia, Argentina); for total anaerobes, Reinforced Clostridia Agar (Britannia, Argentina); for lactobacilli, Mann-Rogosa-Sharpe Agar (MRS Britania, Argentina) and for bifidobacteria, Mann-Rogosa-Sharpe Agar (Britannia, Argentina) plus 5\% of nalidixic acid, neomycin sulphate (Britannia, Argentina) and lithium chloride (Merk, Argentina) solution. Each process was performed per triplicate.

Samples intended for the search of anaerobes, lactobacilli and bifidobacteria were incubated for $48 / 72$ hours at $37^{\circ} \mathrm{C}$ in anaerobiosis jar (GENbox bioMerieux, France). Samples intended to isolate enterobacteria were incubated in aerobio- sis for $24 / 48$ hours at $37^{\circ} \mathrm{C}$. After incubation period, the counting of colony forming units per gram of faeces (CFU / g) was done per each of the bacterial groups studied and quantitative variations according to the diet administrated were compared. Statistical analysis was performed after logarithmic conversion of primary counts of cultures.

Calcium, iron and zinc content from experimental diets, iron and zinc from liver samples as well as calcium and zinc from femur samples were measured by atomic absorption spectrophotometry after a mineralization procedure as previously described [21].

\section{Statistics}

All data were analyzed using a computer statistical program (Graph Pad PRISM®, Version 3.00 1999, GraphPad Software Inc., San Diego CA, USA).

The parameters statistically analyzed were: initial and final body weight, average growth rate, liver and femur weight, iron and zinc liver content as well as calcium and zinc femur content. The statistical analysis were performed by one way analysis of variance (ANOVA) for initial and final body weight, average growth rate, femur weight, zinc and iron liver content as well as calcium and zinc femur content. The a posteriori tests performed were TukeyKramer test for femur weight, average growth rate and calcium and zinc femur content and Student- Newman- Keuls test for initial and final body weight and also zinc and iron liver content. Kruskal Wallis test was used to evaluate liver weight.

An ANOVA was also performed to compare the effect of the different treatments at each time on bacterial recount followed by the a posteriori test of Student-Newman- Keuls to evaluate the differences among the groups.

The level of significance was considered as $\mathrm{p}<0.05$ in all tests.

\section{RESULTS}

\section{Mineral Analysis}

Food intake as well as initial and final body weight are shown in Table 3. All treatments were initiated with animals sorted by groups that did not have significant differences ( $p$ $<0.05$ ) on their body weight and after the study with the corresponding treatments, final body weight of all groups did not show significant differences either $(\mathrm{p}<0.05)$, excluding group 1, as might be expected. It can be noticed that group 1 
has the lowest food intake, which had an impact on growth that can be seen on Fig. (1) as an almost plane body weight curve. Groups 2, 3 and 4 showed normal as well as similar food intake (Table 3) due to the fact that their respective feeding diets suited all nutritional recommendations and were administrated to them during the whole treatment period. Therefore, their body weight curves (Fig. 1) were considered normal and were taken into account as control curves for groups 5, 6 and 7 under study, respectively. Groups 5, 6 and 7, that were put to a depletion-repletion treatment, showed values of food intake that were between group 1 and their respective control groups (Table 3). It is worth mentioning that for these groups, higher values for food intake were achieved during the repletion period as it is shown in Table 3. The impact that these results had on body weight can be noticed in Fig. (1), where body weight curves of groups 5, 6 and 7 started almost plane during the depletion period, the same as group's 1 body growth curve. But after the repletion period and regardless of the diet administrated, these groups reached a normal status of body growth, the same as their respective control groups on day 28 of the treatment. The statistical analysis that supported the results described above is shown in Table 3. The results expressed as values for growth rate are also shown on Table 3. Group 1 showed the lowest growth rate along all the treatment period while groups 5, 6 and 7 showed considerably low growth rates during depletion period than groups 2, 3 and 4 but these was reverted during the repletion period since results were higher for groups 5 and 6 than for control groups 2 and 3 respectively. Growth rate for group 7 showed a tendency to be higher than for group 4 during repletion period.

Table 3. Total Food Intake, Initial and Final Body Weight and Average Growth Rate During the Treatment Period with Experimental Diets

\begin{tabular}{|c|c|c|c|c|c|c|c|}
\hline Group & \multicolumn{2}{|c|}{ Treatment } & $\begin{array}{l}\text { Total Food } \\
\text { Intake (g) }\end{array}$ & $\begin{array}{l}\text { Total Food Intake for Each } \\
\text { Treatment Period (g) }\end{array}$ & \multicolumn{2}{|c|}{ Body Weight (g) } & $\begin{array}{l}\text { Average Growth } \\
\text { Rate (g/day) }\end{array}$ \\
\hline 1 & $\mathrm{D}$ & 28 & 2788 & 2788 & $46.9 \pm 9.3^{\mathrm{a}}$ & $79.9 \pm 14.7^{\mathrm{b}}$ & $1.18^{\mathrm{a}}$ \\
\hline 2 & NS & 28 & 4083 & 4083 & $50.4 \pm 7.5^{\mathrm{a}}$ & $134.7 \pm 9.9^{\mathrm{c}}$ & $3.02^{\mathrm{bd}}$ \\
\hline 3 & $\mathrm{NE}$ & 28 & 4063 & 4063 & $48.9 \pm 4.4^{\mathrm{a}}$ & $129.2 \pm 11.6^{\mathrm{c}}$ & $2.85^{\mathrm{b}}$ \\
\hline \multirow{2}{*}{5} & D & 14 & \multirow{2}{*}{3611} & 1323 & \multirow{2}{*}{$51.0 \pm 9.3^{\mathrm{a}}$} & \multirow{2}{*}{$130.6 \pm 10.7^{\mathrm{c}}$} & $1.36^{\mathrm{a}}$ \\
\hline & NS & 14 & & 2288 & & & $4.29^{\mathrm{c}}$ \\
\hline \multirow{2}{*}{6} & D & 14 & \multirow{2}{*}{3419} & 1185 & \multirow{2}{*}{$44.5 \pm 2.0^{\mathrm{a}}$} & \multirow{2}{*}{$125.8 \pm 6.4^{\mathrm{c}}$} & $1.31^{\mathrm{a}}$ \\
\hline & $\mathrm{NE}$ & 14 & & 2234 & & & $4.51^{\mathrm{c}}$ \\
\hline
\end{tabular}

Values with different superscript for average growth rate and initial and final body weight have significant differences ( $\mathrm{p}<0.05$ ). Results for initial and final body weight are shown as mean $\pm \mathrm{SD}$ and the rest are shown only as means. Values are shown as mean $\pm \mathrm{SD}$.

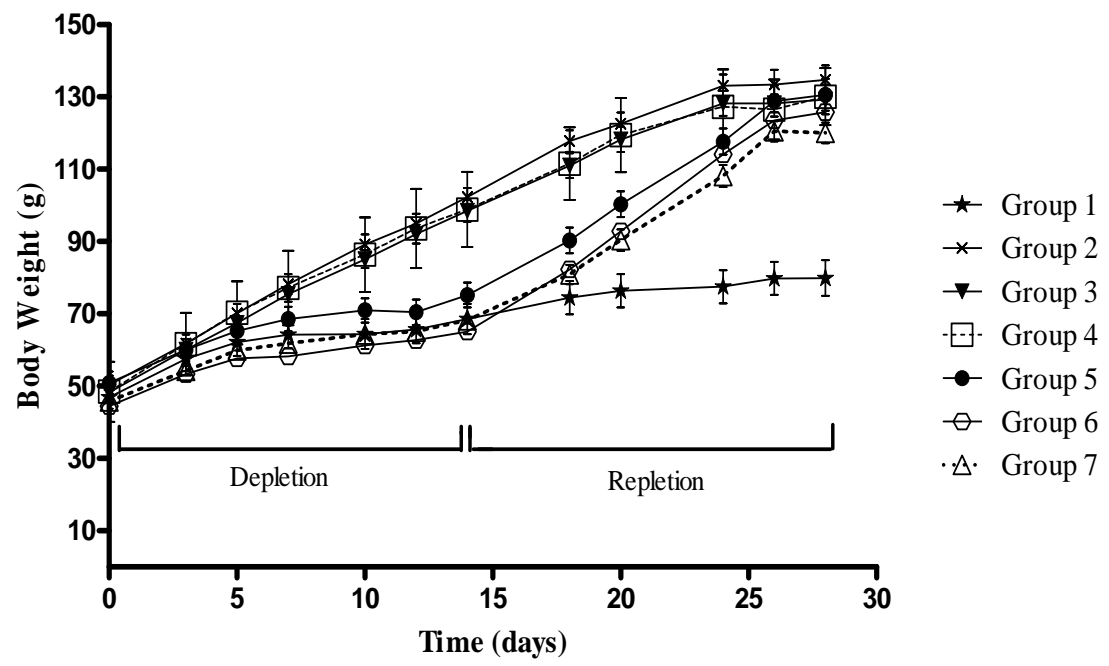

Fig. (1). Growth curves for groups under treatment with experimental diets. Values are shown as mean $\pm \mathrm{SD}$. 
Table 4 shows the results of mineral intake for each group during the treatment period with experimental diets. Group 1 showed the lowest mineral intake values during the treatment period. Groups 5, 6 and 7 showed considerably low mineral intake values during the depletion period compared to groups 2, 3 and 4 but these start to revert during the repletion period though total mineral intake remain lower than for control groups. All these values were the consequence not only of the mineral composition of the experimental diets but also of the food intake for each group under study. That is, a lower mineral intake for the three minerals evaluated (calcium, iron and zinc) was observed because of the consumption of deficient diets but also because the total food intake of groups 1 , 5,6 , and 7 fell down as was mentioned and shown above.

The results concerning the status of bone and liver mineral deposits are shown in Table 5. Liver weight as well as zinc and iron liver content showed no significant differences among the groups under study. However, femur weight as well as zinc and calcium femur content were different among groups. The lowest values for all these parameters were for animals in group 1. Femur weight was higher for groups 2, 3 and 4 as expected for control groups, and lower for group 1 as expected for a deficient group. Groups 5, 6 and 7 showed values that were between group 1 and control groups (2, 3 and 4). With regard to calcium femur content, it was higher for groups 3 and 4 than for group 2 among controls and was also higher for groups 6 and 7 among groups submitted to the depletion-repletion treatment. It is noticeable that groups $3,4,6$ and 7 received diets containing different mineral sources than groups 2 and 5 and calcium femur content resulted higher for those groups fed with diets containing the mineral sources under study (groups 3, 4, 6 and 7) than for those which received diets with standard mineral sources (groups 2 and 5) regardless of the presence or absence of the prebiotic. The zinc femur content also showed interesting results. Values of groups 4 and 7 were the highest observed which resulted statistically different from that of groups 1,2 and 5 and showed a tendency to be higher than those of groups 3 and 6 which received diets containing the same mineral sources but without the prebiotic added as in diets received by groups 4 and 7 . These results suggested that the prebiotic under study may have a positive effect in the absorption or bone deposition of zinc.

\section{Bacteriological Analysis}

Total enterobacteria recount did not register significant changes. Some batches may have shown a slight decrease but it was not significant (less than one logarithm in group 4 after 28 days of experimental diet). Total anaerobic bacteria recount remain constant during the whole experience. In the same way, lactobacilli isolated concentrations remained constant throughout the experiment (data not shown).

However, bifidobacteria recount is significantly increased for group 4 (animals fed with combined mix diet containing the mineral sources under study and the prebiotic) one logarithm as it can be noticed on Fig. (2). This increase was determined at 14 days of the treatment with experimental diets and was maintained until day 28 and also 48 hours and 10 days after the interruption of the treatment (see Fig. 3). Group 7 (animals submitted to a depletion period and then repleted with the same diet fed to group 4), showed the same behavior than group 4 after the repletion. Nevertheless, the bifidobacteria recount decreased after the end of the treatment, that is after day 28 (see Fig. 4).

\section{DISCUSSION AND CONCLUSION}

An insufficient mineral intake still represents one of the most relevant causes of growth impairment. Thus, it is evident the importance of consuming a diet with an adequate mineral content in order to prevent or eliminate the severe consequences associated with their deficiency. Prebiotics can be associated with this since they have been recently described and analyzed as potential beneficial factors for mineral absorption. Some authors have reported positive effects on the absorption of several nutrients such as calcium, magnesium, iron, zinc and copper when a prebiotic of the fructooligosaccharide type was added to the diet [22].

Table 4. Calcium, Iron and Zinc Intake During the Treatment Period with Experimental Diets

\begin{tabular}{|c|c|c|c|c|c|}
\hline Group & Diet & Period (Days) & $\mathrm{Ca}(\mathrm{g})$ & $\mathrm{Fe}(\mathrm{mg})$ & $\mathrm{Zn}$ (mg) \\
\hline 2 & NS & 28 & 22.9 & 142.9 & 123.3 \\
\hline \multirow[t]{2}{*}{5} & $\mathrm{D}$ & 14 & 0.2 & 7.5 & 8.1 \\
\hline & NS & 14 & 12.8 & 80.1 & 69.1 \\
\hline 6 & $\mathrm{D}$ & 14 & 0.2 & 6.8 & 7.2 \\
\hline \multirow[t]{2}{*}{7} & $\mathrm{D}$ & 14 & 0.2 & 5.6 & 6.0 \\
\hline & NEP & 14 & 10.2 & 62.1 & 61,7 \\
\hline
\end{tabular}


Table 5. Bone and Liver Mineral Content of Groups Under Study

\begin{tabular}{|c|c|c|c|c|c|c|}
\hline \multirow[b]{2}{*}{ Group } & \multicolumn{6}{|c|}{ Deposit Mineral Content (mg/Kg) } \\
\hline & \multicolumn{3}{|c|}{ Liver } & \multicolumn{3}{|c|}{ Femur } \\
\hline 1 & $8.19 \pm 0.51$ & $157.6 \pm 18.3$ & $36.2 \pm 4.7$ & $0.552 \pm 0.043^{\mathrm{a}}$ & $64871 \pm 8005^{\mathrm{a}}$ & $73 \pm 8^{\mathrm{a}}$ \\
\hline 3 & $8.11 \pm 0.79$ & $154.7 \pm 21.9$ & $31.9 \pm 2.2$ & $0.649 \pm 0.071^{\mathrm{b}}$ & $101600 \pm 8363^{c}$ & $120 \pm 9^{\mathrm{bc}}$ \\
\hline 4 & $8.22 \pm 0.46$ & $154.7 \pm 26.7$ & $35 . \pm 8.8$ & $0.667 \pm 0.061^{\mathrm{b}}$ & $101992 \pm 8195^{\mathrm{c}}$ & $124 \pm 7^{\mathrm{c}}$ \\
\hline 5 & $6.74 \pm 2.46$ & $156.3 \pm 15.0$ & $36.6 \pm 3.8$ & $0.628 \pm 0.062^{\mathrm{ab}}$ & $90804 \pm 7106^{\mathrm{b}}$ & $112 \pm 11^{\mathrm{b}}$ \\
\hline 6 & $7.88 \pm 3.15$ & $132.2 \pm 15.0$ & $29.5 \pm 2.0$ & $0.604 \pm 0.024^{\mathrm{ab}}$ & $106477 \pm 4467^{\mathrm{c}}$ & $121 \pm 6^{\text {bc }}$ \\
\hline
\end{tabular}

Bone and liver mineral content of groups under study. Values are shown as mean \pm SD and those with different superscript in a column have significant differences ( $<<0.05$ ).

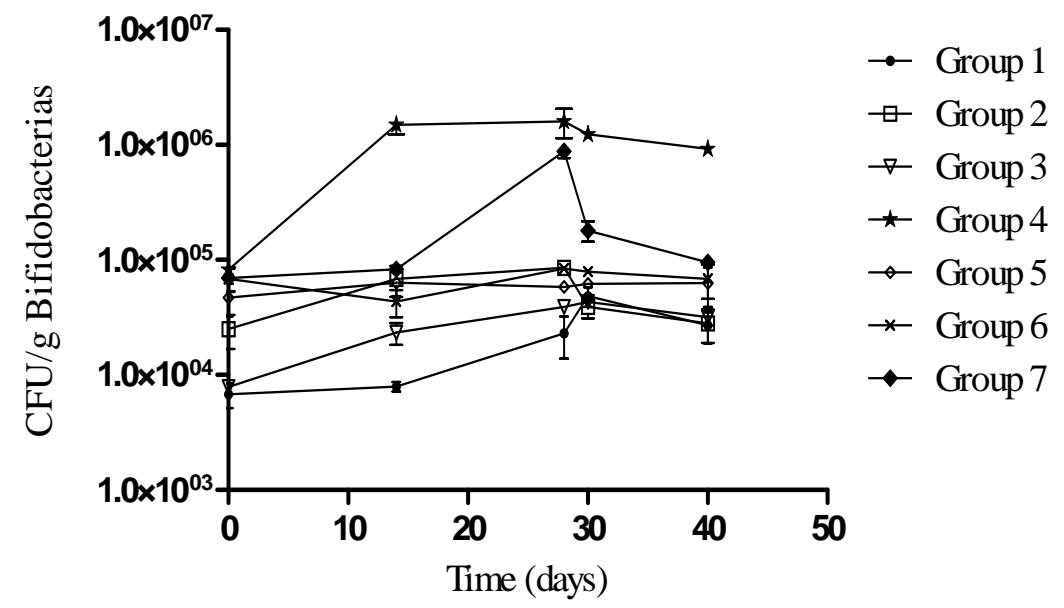

Fig. (2). Bifidobacteria recount for each group under treatment. Values are shown as mean \pm SD.

Our results showed that the combined mineral mix composed by calcium, iron and zinc as well as a prebiotic, has positive effects on growth and intestinal microflora of growing animals that consumed it as part of their diet. Moreover, it has also a positive effect in animals recovering from a period of nutritional depletion.

Mineral deficiency, particularly of calcium, iron and zinc, resulted in deleterious effects on animal body growth and appetite. However this situation could be reverted with a normal diet on its mineral content as demonstrated with this study. That is, despite some groups were submitted to a depletion-repletion period, they were able to restore their body growth status and also reach comparable values of mineral deposit with those presented by control groups. The effects of a diet containing the combined mineral mix under study on body growth showed that mineral sources under study included in its composition had the same physiologic behavior than those of reference used for comparative purposes. Thus, all experimental groups, except for that which received the deficient diet during all the study, achieved the same final body weight and even those previously submitted to a depletion period were able to recover a normal body growth status at the end of the treatment despite the mineral sources used, as already mentioned.

Mineral deposits evaluated as zinc and calcium bone content and as zinc and iron liver content, were comparable with those reported by other authors [23-25] and confirmed the results mentioned above. The mineral sources under study behaved like the reference standards in terms of the organ weight or the organ mineral content. In the case of liver, all groups showed similar values as expected by iron and zinc homeostasis in liver as a rapid interchangeable pool that is highly susceptible to changes in mineral dietary intake [26]. Therefore, the adequacy of the mineral content of the diets supplied by the end of the study to the nutritional recommendations might have been enough to provoke that no differences could be observed among groups for this deposit. In the case of bone, no differences were appreciated in femur weight for groups receiving the combined mix with mineral sources under study (with or without the addition of the pre- 


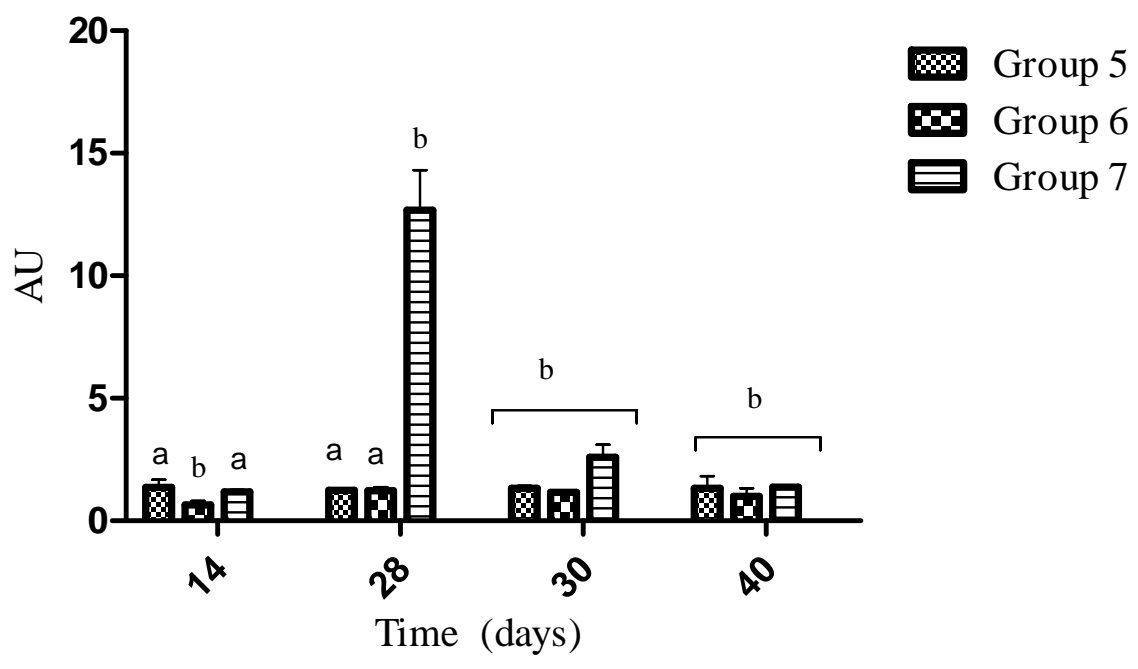

Fig. (3). Bifidobacteria recount increase. Results were calculated as a ratio between $\mathrm{CFU} / \mathrm{g}$ on a given day and the same for day 0 . Values are shown in arbitrary units $(\mathrm{AU}=[\mathrm{CFU} / \mathrm{g}]$ for a given day $/[\mathrm{CFU} / \mathrm{g}]$ for day 0$)$ for each group under treatment. The days evaluated were 14,28 , 30 and 40. Results are expressed as mean \pm SD and bars not sharing a superscript are significantly different.

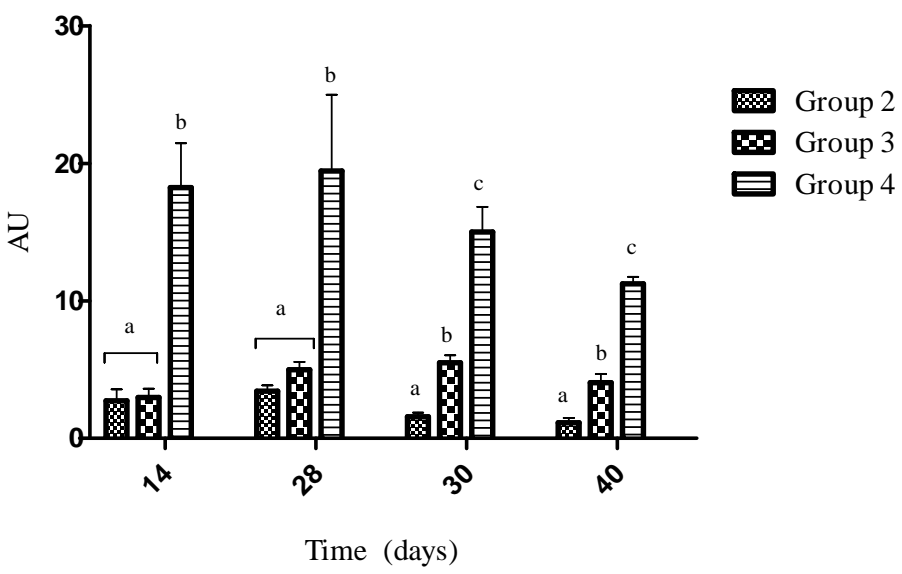

Fig. (4). Bifidobacteria recount increase for groups submitted to a depletion repletion treatment. Results were calculated as a ratio between CFU/g on a given day and the same for day 0 . Values are shown in arbitrary units $(\mathrm{AU}=[\mathrm{CFU} / \mathrm{g}]$ for a given day/[CFU/g] for day 0$)$ for each group under treatment. The days evaluated were 14, 28, 30 and 40. Results are expressed as mean \pm SD and bars not sharing a superscript are significantly different.

biotic) compared to those receiving the standard ones. However, results showed a noticeable tendency of increased zinc femur deposition for animals receiving the combined mineral mix under evaluation. Had the treatment been longer, these differences might have been observed statistically and not just as a tendency. It is important to notice that calcium femur content was higher for groups receiving diets containing the sources under study compared to those groups fed with diets containing reference sources. This means that there may be a positive effect related to the mineral sources evaluated that enhance calcium absorption or its bone deposition. On the present work, the effect of this combined mix, particularly of it's prebiotic on mineral deposition, can be suggested for zinc but not for calcium [27] although many reports have referenced it $[12,13]$.

The mechanisms related with these increments in mineral absorption are not fully studied but several are proposed that may explain these effects. The most common cited is the greater surface resorption developed due to the enterocytes proliferation as a consequence of the presence of bacterial fermentation products (mainly lactate and butyrate) $[22,28$, 29]. Some other morphologic and physiologic changes could also be induced in the intestine such as an increase in the crypts height and the vein flow that may as well be related to the enhanced mineral absorption [28]. Other causes of these effects are the increment in solubilization of several minerals by the presence of short chain fatty acids (SCFA) which have been introduced already as important products of bacterial fermentation. Low $\mathrm{pH}$ caused by SCFA is also a factor that could increase mineral solubility in the gut lumen [22, $29,30]$. An increment in the expression of calcium binding proteins or in calcium transport by a non saturable paracellular pathway could also lead to this mineral's better absorption $[22,28,29]$.

It is important to consider that children fed with breast milk have an increased quantity of intestinal bifidobacteria but the beneficial effects that they produce are combined with the extraordinary characteristics that such food accounts 
for [31, 32], so bifidobacteria in infants provides mainly a prevention against many enteropathogens. It is also important to consider that in adulthood and elderly, the amount of bifidobacteria decreases considerably. Taking that into account, search and confirmation of bifidogenic effects in certain substances is a valuable tool to supplement diets that could provide this function.

Many studies have demonstrated the stimulatory effect of prebiotics on lactobacilli and bifidobacteria [33-35] development, due to the immunochemical mechanisms produced by the fermentation process. Prebiotic fibers are mainly oligosaccharides, inulin and lactulose and they have shown to have bifidogenic effect on colon. The present work confirms that prebiotics have such effect.

An interesting fact is the difference observed in the bifidobacteria population for the group that received a diet containing the prebiotic during the whole treatment and the group which received the diet with the prebiotic after being submitted to a depletion period. Thus, the time of consumption of the prebiotic is an important parameter to evaluate and it has to be taken into consideration when choosing the vehicle that will be fortified. That is, for this mineral mix containing a prebiotic to be successful and achieve its gold, it will have to be consumed on a daily basis. Milks as well as yoghurts are excellent vehicles examples because they suit this requirement.

Mineral food fortification remains an interesting and effective strategy to overcome mineral deficiency and malnutrition. In the particular case of the combined mix evaluated on the present work, the addition of a component such as a prebiotic might enhance the positive effects reached for mineral supplementation and also prevent other affections since it has beneficial effects on its own. This also means that the target people who would consume a fortified food of these characteristics might be from different ages. However, there are still more trials that remain to be done as to confirm the technological advantages of this combined mix over potential changes on the organoleptic characteristics of the vehicle used or even evaluate other vehicles to assure the possibility of other food supplementation. This last concept could be an interesting matter to increase its versatility.

The analysis of the effects that the mineral mix has on body growth as well as on mineral deposits suggests that mineral sources under study present some advantages compared to the reference standard sources. Furthermore, its combination with a prebiotic may produce some other positive effects related to mineral absorption and intestinal microflora composition.

\section{ABREVIATIONS}

$\mathrm{D}=$ Deficient diets

NS $=$ Normal diet on its mineral content with $\mathrm{Ca}$, $\mathrm{Fe}$ and $\mathrm{Zn}$ reference sources

$\mathrm{NE}=$ Normal diet on its mineral content with $\mathrm{Ca}$, $\mathrm{Fe}$ and $\mathrm{Zn}$ evaluated sources

NEP $=$ Normal diet on its mineral content with $\mathrm{Ca}$, $\mathrm{Fe}$ and $\mathrm{Zn}$ evaluated sources and the addition of a prebiotic
$\mathrm{AU}=$ Arbitrary units

$\mathrm{CFU}=$ Colony forming units

ANOVA $=$ Analysis of variance

\section{REFERENCES}

[1] Anderson JJ, Garner SC. Calcium and phosphorus in health and disease. CRC Press; 1995.

[2] Mataix Verdú J. Nutrientes y sus funciones. In: Serra Majem L, Aranceta Bartrina J, Eds. Nutrición y Salud pública: métodos bases científicas y aplicaciones. España: Elsevier, 2006: pp. 8-19.

[3] Salgueiro J, Zubillaga M, Lysionek A, et al. Zinc as an essential micronutrient: a review. Nutr Res 2000; 20(5): 737-55.

[4] Salgueiro J, Weill R, Zubillaga M, et al. Zinc deficiency and growth. Biol Trace Element Res 2004; 99: 49-69.

[5] Frederickson CJ, Suh SW, Silva D, Frederickson CJ, Thompson $\mathrm{RB}$. Importance of zinc in the central nervous system: The zinccontaining neuron. J Nutr 2000; 130: 1471S-83S.

[6] Cheryan M. Phytic acid interactions in food systems. Crit Rev Food Sci Nutr 1980; 13(4): 297-335.

[7] Salgueiro J, Leonardi N, Zubillaga M, et al. Normal growth rate in rats is recovered after a period of zinc deficiency by restoration of Zinc supply by means of a Zinc-fortified petit Suisse cheese. Biol Trace Elem Res 2004; 104(3): 269-73.

[8] Sandstead HH, Penland JG, Alcock NW, et al. Effects of repletion with zinc and other micronutrients on neuropsychologic performance and growth of Chinese children. Am J Clin Nutr 1998; 68: 470S-5S.

[9] Giugliano R, Millward DJ. Growth and Zinc homeostasis in the severely Zn-deficient rat. Br J Nutr 1984; 52: 545-60.

[10] Gibson RS, Ferguson EL. Nutrition intervention strategies to combat zinc deficiency in developing countries. Nutr Res Rev 1998; 11: 115-31.

[11] Salgueiro MJ, Zubillaga M, Lysionek A, Caro R, Weill R, Boccio J. Fortification strategies to combat zinc and iron deficiency. Nutr Rev 2002; 60(2): 52-8.

[12] Cashman K. Prebiotics and calcium bioavailability. In Tannock GW, Ed. Probiotics and prebiotics: where are we going? Wymondham: Caister Academic Press, 2002; pp. 149-74.

[13] Coxam V. Current Data with inulin-type Fructans and Calcium, Targeting Bone Health in Adults. J Nutr 2007; 137: 2527S- 33S.

[14] Aybar M, Grau A, Sanchez RA, Sanchez S. Hypoglycemic effect of the water extract of Smallantus sonchifolius (yacon) leaves in normal and diabetic rats. J Ethnopharmacol 2001; 74: 125-32.

[15] Delzenne NM, Kok N. Effects of fructans-type prebiotics on lipid metabolism. Am J Clin Nutr 2001; 73: 456S-8S

[16] Marti del MA, Moreno-Aliaga MJ, Marinez JA. Efecto de los prebióticos sobre el metabolismo lipídico. Nutr Hosp 2003; 18(4): 181-8.

[17] Scheppach W. Effects of short chain fatty acids on gut morphology and function. Gut 1994; 1: S35-S8.

[18] Hamilton-Miller JMT. Probiotics and prebiotics in the elderly. Postgrad Med J 2004; 80: 447-51.

[19] Jones P. Clinical nutrition: 7. Functional foods- more than just nutrition. Can Med Assoc J 2002; 166(12): 1555-63.

[20] Reeves PG, Nielsen FH, Fahey Jr. GC. AIN-93 purified diets for laboratory rodents: Final report of the american institute of nutrition ad hoc writing committee on the reformulation of the AIN-76A rodent diet. J Nutr 1993; 123: 1939-51.

[21] Lysionek AE, Zubillaga MB, Calmanovici GP, et al. Modification of the ferrozine technique to analyze iron contents in different foods. Biol Trace Elem Res 1998; 65: 87-94.

[22] Delzenne N, Aertssens J, Verplaetse H, Roccaro M, Roberfroid M. Effect of fermentable fructo- oligosaccharides on mineral, nitrogen and energy digestive balance in the rat. Life Sci 1995; 57(17): 1579-87.

[23] Prasad AS, Oberleas D, Wolf P, Horwitz JP. Studies on zinc deficiency; changes in trace elements and enzyme activities in tissues of zinc- deficient rats. J Clin Invest 1967; 46(4): 549-57.

[24] Kang HK, Harvey PW, Valetine JL, Swendseid ME. Zinc, iron, copper, and magnesium concentrations in tissues of rats fed various amounts of zinc. Clin Chem 1977; 23(10): 1834-7. 
[25] Tesan FC, Collia N, Arnoldi S, et al. Relative bioavailability of zinc in yogurt using body weight gain, femur weight and bone zinc content in rats as markers. Open Nutra J 2009; 2: 16-9.

[26] Graham RH, Chua ACG, Herbison CE, Olynyk JK, Trinder D. Liver iron intake. World J Gastroenterol 2007; 13(35): 472536.

[27] Jamieson JA, Ryz NR, Taylor CG, Weiler HA. Dietary long-chain inulin reduces abdominal fat but has no effect on bone density in growing female rats. Br J Nutr 2008; 100(2): 451-9.

[28] Scholz-Ahrens KE, Schaafsma G, Van den Heuvel EG, Schrezenmeir J. Effects of prebiotics on mineral metabolism. Am J Clin Nutr 2001; 73: 459S- 64S.

[29] Scholz-Ahrens KE, Ade P, Berit M, Weber P, Wolfram T, Yahya A. Prebiotics, Probiotics and Symbiotics Affect Mineral Absorption, Bone mineral Content, and Bone Structure. J Nutr 2007; 137: $838 \mathrm{~S}-46 \mathrm{~S}$.
[30] Scholz-Ahrens KE, Schrezenmeir J. Inulin, oligofructose and mineral metabolism- experimental data and mechanism. Br J Nutr 2002; 87(2): S179-S86.

[31] Akre J. Inmunological Qualities Of Breast Milk. In: Infant Feeding: The Physiological Basis. Bull World Health Organ 1989; 67: 314.

[32] Riveron CR. Value of Breast milk immune. Rev Cubana Pediatr 1998; 67(2).

[33] Ruperez P. Oligosacáridos bifidogénicos. Food Sci Technol Int 1998; 4: 237-43.

[34] Raish O, Leplingard A, Mater D, et al. Survival of lactobacillus casei in the human digestive tract after consumption of fermented milk. Appl Environ Microbiol 2006; 72(8): 5615-7.

[35] Meyer D, Stasse-Wolthuis M. The bifidogenic effect of inulin and oligofructose and its consequences for gut health. Eur J Clin Nutr 2009; 63(11): 1277-89.

(C) Tesan et al.; Licensee Bentham Open.

This is an open access article licensed under the terms of the Creative Commons Attribution Non-Commercial License (http://creativecommons.org/licenses/ by-nc/3.0/) which permits unrestricted, non-commercial use, distribution and reproduction in any medium, provided the work is properly cited. 